\title{
Neurophysiologic Correlates of Side Effects in Normal Subjects Randomized to Venlafaxine or Placebo
}

\author{
Aimee M Hunter*,', Andrew F Leuchter', Melinda L Morgan', lan A Cook', Michelle Abrams', \\ Barbara Siegman', David J DeBrota ${ }^{2}$ and William Z Potter ${ }^{2}$ \\ 'Behavioral Pharmacology Laboratory, Department of Psychiatry and Biobehavioral Sciences, UCLA Neuropsychiatric Institute; David Geffen \\ School of Medicine, Los Angeles, CA, USA; ${ }^{2}$ Lilly Research Laboratories, Department of Psychiatry and Biobehavioral Sciences, UCLA \\ Neuropsychiatric Institute; David Geffen School of Medicine, Los Angeles, CA, USA
}

\begin{abstract}
Adverse events reported in the context of medication administration may be due to pharmacodynamic and/or nonpharmacodynamic effects (eg, nocebo phenomena). Neurophysiological substrates of side effects may be examined in placebo-controlled antidepressant treatment trials. We explored the relationship between side effects and regional neurophysiologic changes in normal subjects receiving a I-week placebo lead-in followed by 4 weeks randomized treatment with placebo $(n=15)$ or venlafaxine IR $(n=17)$. Quantitative electroencephalographic (QEEG) cordance measures were obtained before and during treatment, and side effects were assessed weekly using semistructured interviews. Side effect burden, characterized as the mean number of side effects per postrandomization visit, correlated significantly with neurophysiologic changes in the antidepressant group but not the placebo group. Medication group side effects were negatively correlated with changes in prefrontal cordance at end of placebo lead-in $(r=-0.67, p<0.003)$, at 2 weeks $(r=-0.77, p<0.002)$, and at 4 weeks $(r=-0.77, p<0.004)$ post randomization. After controlling for the prefrontal change at the end of placebo lead-in, postrandomization brain changes did not further explain side effect burden. Changes in prefrontal brain function associated with later antidepressant side effects were observed during placebo lead-in-prior to the administration of medication. Prefrontal brain function during brief placebo administration may help explain susceptibility to the development of antidepressant side effects. Results of these exploratory hypothesis-generating analyses should be considered tentative until replicated.
\end{abstract}

Neuropsychopharmacology (2005) 30, 792-799, advance online publication, 22 December 2004; doi: 10.1038/sj.npp. 1300652

Keywords: placebo effect; side effects; antidepressant medication; EEG; cordance; randomized controlled trial

\section{INTRODUCTION}

Antidepressant medication side effects pose an important and widespread medical concern. Despite the development of medications with increasingly selective pharmacologic profiles, many patients suffer discomfort or terminate antidepressant treatment because of adverse effects. A recent survey in primary care found selective serotonin reuptake inhibitors (SSRIs) to be the class of drug most frequently involved in adverse drug events (Gandhi et al, 2003). Antidepressant side effects are associated with decreased quality of life (Barge-Schaapveld and Nicolson, 2002) and are frequently cited as the reason for discontinuation of pharmacotherapy across medication classes

*Correspondence: Dr AM Hunter, UCLA Neuropsychiatric Institute, 760 Westwood Plaza, Rm. 37-359, Los Angeles, CA 90024-1759, USA, Tel: + 310206 2237, Fax: + 310825 7642,

E-mail: amhunter@ucla.edu

Received 24 May 2004; revised 15 October 2004; accepted 12 November 2004

Online publication: 17 November 2004 at http://www.acnp.org/citations/ Npp I I 1704040244/default.pdf
(Anderson and Tomenson 1995; Montgomery and Kasper 1995; Montgomery et al, 1994).

Adverse effects associated with administration of antidepressant medication may be determined by a multitude of pharmacodynamic and/or nonpharmacodynamic factors (Barsky et al, 2002). Pharmacodynamic effects may be anticipated on the basis of receptor binding profiles. Recent evidence also suggests that some medication-specific side effects may be related to genetic polymorphisms governing drug metabolism (Yoshida et al, 2003; Lessard et al, 1999; Roberts et al, 2002) or receptor function (Greer et al, 2003). However, nonpharmacodynamic factors, such as expectation alone, also can trigger distressing symptoms - an effect termed the 'nocebo' phenomenon (Kennedy 1961; Hahn 1997). Patients' expectations of side effects may arise from a variety of sources including: information contained in the medication package insert, specific inquiries about side effects posed by the treating physician or nurse, informed word-of-mouth or internet-based sources, information provided in consent forms describing potential adverse effects in the clinical trial setting (Myers et al, 1987), or classical conditioning effects of prior experience with medication. Given that medication and expectation may 
produce similar adverse effects and these factors can operate simultaneously, it is difficult to determine the 'cause' of side effects reported in the clinical setting.

One method to distinguish between side effects that are induced by pharmacodynamic vs nonpharmacodynamic factors may be to examine brain functional changes associated with side effects reported during treatment with antidepressant medication $v s$ placebo. During treatment with placebo, side effects could arise only from nonpharmacodynamic factors, whereas during treatment with medication, side effects could be due to pharmacodynamic factors, nonpharmacodynamic factors, or their confluence. Other studies have compared brain functional changes associated with clinical response to antidepressant medication vs placebo in major depressive disorder (MDD) (Leuchter et al, 2002; Mayberg et al, 2002); however, none has addressed the relationship between brain function and side effects during treatment with medication or placebo.

Administration of antidepressants to normal healthy subjects offers a particularly helpful paradigm for understanding neurophysiologic changes associated with antidepressant-related side effects. Because normal subjects have no current or past depression, nor exposure to antidepressants, administration of antidepressants to normal subjects makes it possible to examine the pharmacologic effects of medication without the confounds of illness or change in illness state. In patients with depressive disorders, for example, changes in brain function observed during treatment could be related to changes in depressive symptoms. Illness status also could introduce a confound in assessing antidepressant side effects because depressed subjects are more susceptible to a variety of somatic complaints including pain, gastrointestinal distress, and somatic anxiety symptoms such as palpitations (Ohayon and Schatzberg, 2003). Another advantage to studying normal subjects is that the observed and reported effects of medication are not confounded by a therapeutic relationship, but instead are shaped more by the pharmacodynamic action of the medication as well as by subjects' general expectations about the medication effect. By examining normal subjects during blinded treatment with antidepressant $v s$ placebo, it is possible to contrast neurophysiologic correlates of side effects that occur in the presence $v s$ absence of drug without the confounds related to illness.

Here we sought to characterize neurophysiological changes associated with side effect burden experienced during treatment with antidepressant medication $v s$ placebo in normal subjects. Data were obtained from a primary study from our laboratory using quantitative electroencephalographic (QEEG) cordance to examine brain function at various pre- and postrandomization time-points in subjects who received a 1-week placebo lead-in and then were randomized to venlafaxine IR or placebo.

\section{SUBJECTS, MATERIALS, AND METHODS}

\section{Participants}

Of 46 individuals screened for eligibility, we enrolled 32 healthy, never-depressed subjects (17 females; 15 males) with a mean age of 36.5 years $(S D=13.2$; range $18-64)$ and a mean education of 14.9 years $(\mathrm{SD}=1.9$; range $12-18)$. Subjects were recruited from community advertisement and were paid for their participation. Screening consisted of a standard clinical evaluation, a structured clinical interview using the Structured Clinical Interview for Axis I DSM-IV Disorders-Patient Edition (SCID-I/P, version 2.0; First et al, 1994), and Hamilton Depression Rating Scale (HAMD; Hamilton, 1960) assessments. All subjects were free of any illness known to affect brain function or which contraindicated the use of venlafaxine; subjects with high blood pressure, heart disease, renal impairment, or cirrhosis of the liver were excluded from participation in the study. We excluded subjects meeting DSM-IV axis I criteria for a current or past mood, anxiety, cognitive, or psychotic disorder on the basis of the SCID-I/P interview, as well as those meeting criteria for cluster A or B axis II diagnoses by clinical interview. No subject ever had received antidepressant medication. Subjects were required to abstain from use of any primarily CNS-active medications including sedative-hypnotics, or other medications with significant CNS activity for ten days prior to entering the study and during the course of the study. Urine toxicology screens were performed to rule out use of psychoactive medications. Subjects were permitted to receive concomitant treatment with medications without significant CNS activity.

All subjects were treated in accordance with ethical standards set forth in the 1975 Declaration of Helsinki. Experimental procedures were explained fully to the subjects and written informed consent was obtained prior to their inclusion in the study. The consent form listed possible side effects as indicated on the manufacturer's package insert. The UCLA Institutional Review Board approved all experimental procedures.

\section{Experimental Design and Procedures}

All subjects underwent a 1-week single-blind placebo leadin before randomization to 4 weeks of double-blind treatment with venlafaxine IR $(n=17)$ or placebo $(n=15)$. Subjects received one $37.5 \mathrm{mg}$ capsule of venlafaxine or placebo, with an increase of $37.5 \mathrm{mg}$ every 3 days (added on a b.i.d schedule) until a dose of $150 \mathrm{mg}$ was reached 10 days after randomization. This dosage increase schedule was selected to be consistent with previous studies (Leuchter et al, 2002; Cook et al, 2002).

Study personnel met with subjects at seven time-points over the course of the study: baseline, end of 1-week placebo lead-in, $48 \mathrm{~h}$ after randomization, and 1, 2, 3, and 4 weeks after randomization. At each visit, a research nurse monitored health status and recorded treatment-emergent symptoms. EEG recordings were obtained at all visits except for week 3.

After data collection, we quantified side effect burden and evaluated the relationship between side effect burden and brain functional changes from baseline at all pre- and postrandomization EEG time-points in medication and placebo groups.

\section{QEEG Techniques}

EEG recordings were performed using the QND system (Neurodata, Inc., Pasadena, CA) while subjects rested in the 
eyes-closed, maximally alert state in a sound-attenuated room with subdued lighting, using procedures previously described in detail (Cook et al, 1998b, 1999; Leuchter et al, 1992). Electrodes were placed with an electrode cap (ElectroCap; Eaton, $\mathrm{OH}$ ) using an extended International 10-20 System with 35 recording electrodes (Figure 1; Cook et al, 1998b). Eye movements were monitored with right infraorbital and left outer canthus electrodes. Data were collected using a $\mathrm{Pz}$ reference montage and were digitized at $256 \mathrm{samples} / \mathrm{ch}$ annel/s, with a high-frequency filter of $70 \mathrm{~Hz}$ and a low-frequency filter of $0.3 \mathrm{~Hz}$. During recording, an EEG technologist who was blinded to treatment condition initially selected approximately $1 \mathrm{~min}$ of artifact-free data. Another technologist, blinded to subject identity and treatment condition, reviewed the data for eye movement, muscle, or other artifacts and made a final selection the first $20-32 \mathrm{~s}$ of artifact-free data for processing. This amount of data has been used to obtain reliable frequency spectra (Leuchter et al, 1992, 1999; Brenner et al, 1995). Data were reformatted by amplitude subtraction to construct a linkedears reference. A fast Fourier transform was used to calculate absolute power (the intensity of energy in a frequency band in microvolts squared) in each of four frequency bands $(0.5-4,4-8,8-12$, and $12-20 \mathrm{~Hz})$.

\section{Cordance Calculations}

Cordance values were calculated for each electrode site in each of the four frequency bands from conventional QEEG absolute and relative power measures, in three steps that are described elsewhere in greater detail (Leuchter et al, 1999). First, EEG power values were computed using a reattributional electrode montage because that montage affords the highest correlation between EEG measures and PET

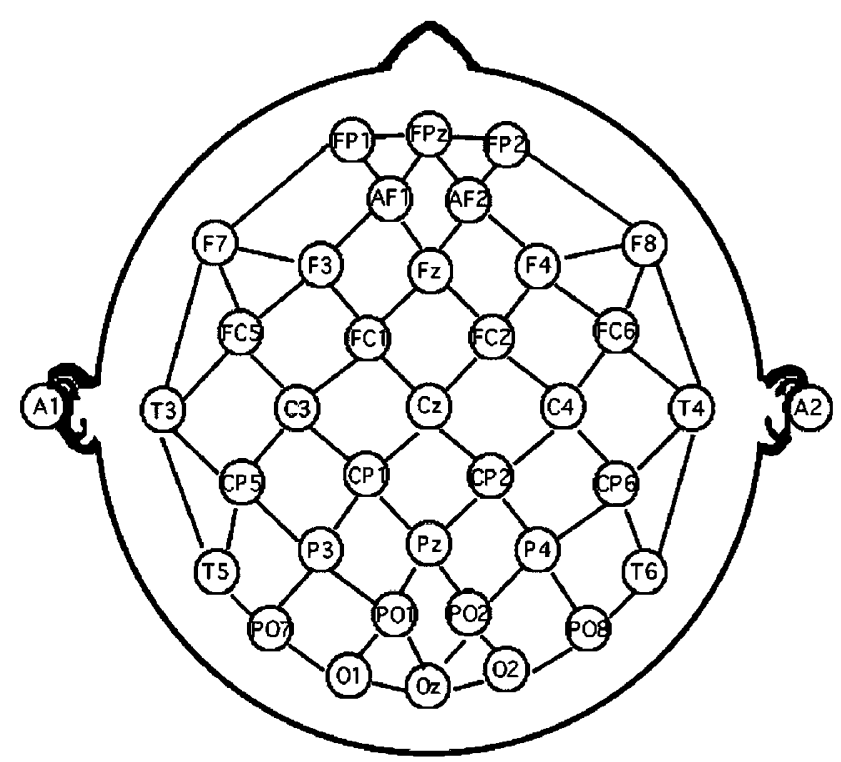

Figure I Electrode montage. Shown are 35 scalp electrodes from the extended International 10-20 System. All electrode pairs sharing a common electrode were averaged to obtain a reattributed power for that electrode. Lines show the bipolar channels used in the reattributional montage. For example, electrode $\mathrm{FPz}$, power was obtained by averaging pairs FPI-FPz, FP2-FPz, AFI-FPz, and AF2-FPz (after Cook et al, 1998). measures of cerebral perfusion. Second, the absolute and relative power values for each electrode site $s$ in each frequency band $f$ in this recording were $z$-transformed to measure deviation from the mean values for that recording, yielding $A_{\text {norm }(s, f)}$ and $R_{\text {norm }(s, f)}$ respectively. Third, these $z$-scores were summed to yield a cordance 'intensity' value, $Z$, for each electrode in each frequency band where $Z_{(s, f)}=A_{\text {norm }(s, f)}+R_{\text {norm }(s, f)}$. We limited our analysis to the theta frequency band $(4-8 \mathrm{~Hz})$ because previous work from this laboratory and others has indicated that energy in the theta band is associated most strongly with action of antidepressant medication (Cook et al, 1998a, 2002; Cook and Leuchter 2001; Ulrich et al 1994). We further limited the number of statistical comparisons by grouping individual electrodes into eight major brain regions: prefrontal (Fp1, Fpz, Fp2), frontocentral (AF1, AF2), central (FC1, Cz, FC2), left temporal (T3, T5), right temporal (T4, T6), left parietal ( $\mathrm{P} 3, \mathrm{CP} 1, \mathrm{CP} 5)$, right parietal (P4, CP2, CP6), and occipital $(\mathrm{O} 1, \mathrm{O} 2, \mathrm{Oz})$ (see Table 1 and Figure 1). Cordance values for electrodes in these groups were averaged together to obtain regional measures.

\section{Side Effect Measures}

A research nurse obtained side effect reports at each visit. Subjects were asked systematically about specific somatic complaints including gastrointestinal upset, cardiovascular disturbance, sleep disturbance, anxiety, and agitation (ie, items that were listed on the consent form as common side effects of venlafaxine IR). Somatic complaints were documented as described by each subject. We later assessed each symptom according to groupings in the Patient Rated Inventory of Side Effects (PRISE; developed for the NIMHfunded STAR-D protocol; Rush, 2001) and tallied the number of symptoms reported at each visit.

Side effect measures were quantified in two ways. First, we used the number of symptoms reported at each timepoint to assess treatment group differences over time. Second, we calculated overall side effect burden exactly once for each subject defined as the mean number of side effects reported per postrandomization visit. This measure enabled us to incorporate both the absolute number of side effects and the persistence of side effects over the trial. This

Table I Brain Regional Electrode Groupings

\begin{tabular}{ll}
\hline Brain region & Electrodes \\
\hline Prefrontal & $\mathrm{FPI}, \mathrm{FP} 2, \mathrm{FPz}$ \\
Central & $\mathrm{FCl}, \mathrm{Cz}, \mathrm{FC} 2$ \\
Frontocentral & $\mathrm{AFI}, \mathrm{AF2}$ \\
Left temporal & $\mathrm{T} 3, \mathrm{~T} 5$ \\
Right temporal & $\mathrm{T} 4, \mathrm{~T} 6$ \\
Left parietal & $\mathrm{P} 3, \mathrm{CPI}, \mathrm{CP5}$ \\
Right parietal & $\mathrm{P} 4, \mathrm{CP} 2, \mathrm{CP} 6$ \\
Occipital & $\mathrm{OI}, \mathrm{O} 2, \mathrm{Oz}$ \\
\hline
\end{tabular}

Electrical activity from electrodes in each group was averaged to obtain brain regional cordance measures. 
measure also allowed us to utilize data from subjects who dropped out prior to the end of the study.

\section{Data Analysis}

Treatment group differences in demographic variables were assessed using Fisher's exact for categorical data (gender), and $t$-tests for continuous variables (age, education). Group differences in baseline regional cordance were assessed using $t$-tests.

We hypothesized that the medication group would report more side effects than the placebo group at every postrandomization visit and we tested the null hypothesis using a repeated measures analysis of variance (ANOVA) with treatment as the between-group factor (venlafaxine or placebo) and time as the within-group factor ( $48 \mathrm{~h}, 1$ week, 2 weeks, 3 weeks, and 4 weeks). We hypothesized also that the medication group would report a greater number of side effects at every postrandomization visit as compared to end of placebo lead-in. Within-group differences in side effects between end of placebo lead-in and each postrandomization visit were evaluated for medication and placebo groups using dependent-samples $t$-tests (one-tailed). All available observations were used.

Change-from-baseline cordance values were calculated for eight brain regions at all EEG time-points: end of placebo lead-in, $48 \mathrm{~h}$, and 1, 2, and 4 weeks after randomization. Pearson's correlation coefficients were used to assess relationships between regional changes in cordance and overall side effect burden (ie, the mean number of side effects per postrandomization visit) for all EEG time-points for medication and placebo groups. At each time-point we used a Bonferroni correction for multiple regional tests (ie, $0.05 / 8$ regions) corrected to $p<0.006$, two-tailed.

After testing regional changes at each time point, those changes found to be significantly associated with side effect burden were analyzed using linear regression with an alpha of $p<0.05$. Regression analysis included only those subjects who completed the trial. Analyses were performed using SPSS software (SPSS, Inc. Chicago, IL).

\section{RESULTS}

All of the subjects who began randomized treatment in the placebo arm $(n=15)$ completed the 5-week trial. In the medication group, four of the 17 subjects who began randomized treatment dropped out of the study prematurely due to adverse events. One subject dropped out after $48 \mathrm{~h}$ on drug and three subjects dropped out after the end of week 1 on drug. QEEG data were unusable for one of the placebo subjects at week 1 and for one of the medication subjects at week 4 . Subjects randomized to venlafaxine $v s$ placebo did not differ statistically with respect to age, gender or education (Table 2).

\section{Mean Side Effects in Venlafaxine and Placebo Groups}

Figure 2 shows mean side effects across time for venlafaxine and placebo groups. No significant group differences were observed at end of placebo lead-in. In the examination of postrandomization side effects, the repeated measures
ANOVA resulted in a significant group effect $\left(\mathrm{F}_{(1,22)}=7.9\right.$, $p=0.005$ ) but no significant effect of time or group by time interaction. Venlafaxine subjects reported a significantly greater number of side effects than did placebo subjects at all postrandomization time-points: $48 \mathrm{~h}$ $\left(t_{(28)}=1.79, p=0.04\right), 1$ week $\left(t_{(27)}=3.11, p=0.002\right), 2$ weeks $\left(t_{(24)}=1.83, p=0.04\right), 3$ weeks $\left(t_{(26)}=1.78, p=0.04\right)$, and 4 weeks $\left(t_{(26)}=2.29, p=0.02\right)$.

Within the venlafaxine group, dependent samples $t$-tests revealed a significantly greater number of side effects at all postrandomization time-points as compared to end of placebo lead-in: $48 \mathrm{~h}\left(t_{(14)}=1.82, p=0.04\right)$, 1 week $\left(t_{(12)}=3.49, p=0.002\right), 2$ weeks $\left(t_{(10)}=2.83, p=0.009\right), 3$ weeks $\left(t_{(11)}=2.11, p=0.03\right)$, and 4 weeks $\left(t_{(11)}=2.03\right.$, $p=0.03$ ). In the placebo group, mean number of side effects did not differ significantly between end of placebo lead-in and any postrandomization time-point.

\section{Brain Function and Side Effect Burden}

Baseline measures of regional cordance did not differ significantly between treatment groups. No significant correlations were found between baseline cordance measures and side effect burden. Although there were trends toward associations between regional cordance measures at baseline and postrandomization side effect burden in the placebo group, these correlations did not reach statistical significance after correcting for multiple comparisons.

Changes from baseline cordance correlated significantly with side effect burden (mean number of side effects per

Table 2 Subject Demographics

\begin{tabular}{lcc}
\hline & Venlafaxine $(\mathbf{n}=\mathbf{I 7})$ & Placebo $(\mathbf{n}=\mathbf{I 5})$ \\
\hline Gender ratio (M:F) & $0.6: 1$ & $1.5: 1$ \\
Age (years \pm SD) & $36.1 \pm 14.5$ & $37.0 \pm 12.0$ \\
Education (years \pm SD) & $14.5 \pm 2.0$ & $15.5 \pm 1.6$ \\
\hline
\end{tabular}

There were no significant demographic differences between subjects randomized to venlafaxine or placebo.

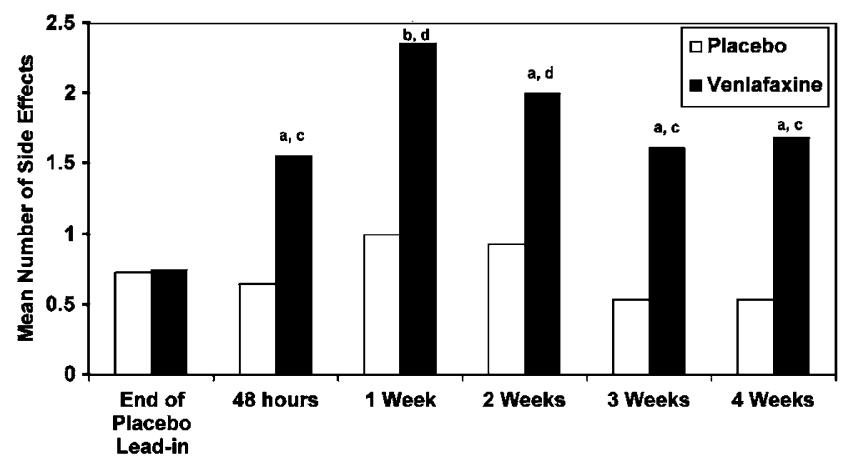

Figure 2 Mean side effects across time for healthy subjects randomized to venlafaxine or placebo. (a) Significantly greater in venlafaxine vs placebo, $p<0.05$. (b) Significantly greater in venlafaxine vs placebo, $p<0.01$. (c) Significantly greater than end of lead-in, $p<0.05$. (d) Significantly greater than end of lead-in, $p<0.01$. 
postrandomization visit) in the venlafaxine group but not the placebo group. Of the eight brain regions examined (Table 1), only changes in the group of channels overlying the prefrontal region (Fp1, Fp2, Fpz; Figure 1) were significantly correlated with antidepressant side effect burden. Side effect burden in the venlafaxine group was significantly associated with decreases in prefrontal cordance occurring at the end of placebo lead-in $(r=-0.67$, $p=0.003)$, and at 2 weeks $(r=-0.77, p=0.002)$, and four weeks $(r=-0.77, p=0.004)$ post randomization. These values were significant at the 0.05 level after correcting for eight regional comparisons per time-point. Mean changes in prefrontal cordance during placebo lead-in for medication (mean $=0.07, \mathrm{SD}=0.86$ ) and placebo $($ mean $=0.06$, $\mathrm{SD}=0.78)$ groups were not statistically different $\left(t_{(31)}\right.$ $=-0.035, p=0.972$ ).

Because medication side effect burden was associated with decreases in prefrontal cordance occurring both during placebo lead-in and after start of medication, we used multiple linear regression analysis to determine the relative contributions of pre- $v s$ postrandomization changes in prefrontal cordance in explaining variance in side effect burden. Specifically, we sought to determine whether the decreases in prefrontal cordance observed during treatment with medication would contribute to the model after accounting for prefrontal change at lead-in. Linear regression using forward stepwise entry revealed that, after entering prefrontal change at end of placebo lead-in, neither the two-week nor the four-week postrandomization correlation entered the model. The model, using only the prefrontal change at end of lead-in, was significant with $\mathrm{R}^{2}=0.58 ; F_{(1,10)}=13.71, p=0.004$. Figure 3 shows topographic changes in cordance at end of placebo lead-in for venlafaxine subjects in the top $(n=5)$ vs bottom $(n=4)$ quartiles of side effect burden.

\section{DISCUSSION}

To our knowledge this is the first report of neurophysiologic changes related to medication side effects, and the first report of an association between neurophysiologic changes during placebo lead-in and a later drug-related outcome.
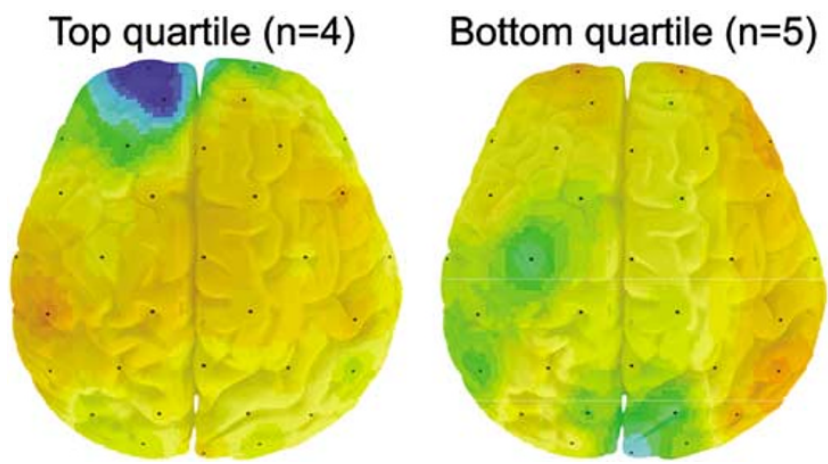

Figure 3 Changes in brain function during placebo lead-in related to later venlafaxine side effects. Maps show changes from baseline cordance at end of placebo lead-in for subjects later falling in the top $(n=4)$ vs bottom quartiles $(n=5)$ of side effect burden during subsequent venlafaxine treatment. Decreases from baseline cordance are in blue; increases are red.
Changes in prefrontal brain function occurring at the end of placebo lead-in, prior to the administration of medication, were associated with later side effect burden in healthy subjects randomized to venlafaxine. Neurophysiologic changes during placebo lead-in were not related to side effects at lead-in; rather, the relationship to side effect burden emerged only after the administration of medication. As such, changes in prefrontal brain function during placebo lead-in appeared to indicate a susceptibility to developing medication side effects.

One question that arises regarding the reports of side effects in these subjects is whether the side effects were 'real,' namely, a pharmacodynamic effect of venlafaxine. In our study, the neurophysiologic changes associated with side effect burden in subjects randomized to venlafaxine appear to be more closely related to nonpharmacodynamic factors than to pharmacodynamic factors. Although postrandomization decreases from baseline prefrontal cordance also were associated with antidepressant side effect burden, brain changes at these time-points did not add explanatory power beyond the change at end of placebo lead-in. The correlations at 2 and 4 weeks after start of drug may have been a continuation of the prefrontal neurophysiologic changes at end of placebo lead-in that correlated with side effects.

One nonpharmacodynamic factor that may contribute to the reporting of adverse events is subject expectations. Experimentally-induced expectations have been shown to produce a wide range of adverse effects including asthmatic symptoms (McFadden et al, 1969), allergic reactions (Jewett et al, 1990), vomiting (Kissel and Barrucand 1964), and hyperalgesia (Benedetti et al, 2003) after receiving an inert treatment. Even when expectations of adverse effects are not explicitly induced, healthy subjects often report side effects. A survey of 109 placebo-controlled medication trials found that $19 \%$ of healthy subjects receiving placebo reported side effects and this figure increased to $28 \%$ with multiple dosing (Rosenzweig et al, 1993). Information provided in consent forms may explain some of the side effects reported by healthy subjects. A placebo-controlled clinical study of aspirin showed a six-fold increase in reports of gastrointestinal upset among patients whose consent forms specifically addressed the possibility of gastrointestinal irritation $v s$ those whose consent forms did not make the reference. In all, $40 \%$ of those reporting gastrointestinal upset were taking placebo (Myers et al, 1987). Classical conditioning also may contribute to subjects' expectations of side effects and may elicit conditioned responses that resemble medication side effects. Pharmaco-conditioning can occur when contextual cues surrounding drug administration come to be associated with the effects of the drug itself (Ader 1997; Siegel, 2002). Among individuals with a history of medication side effects, subsequent medication administration procedures and associated stimuli may elicit conditioned responses in the form of similar adverse effects.

Other studies have shown that pretreatment expectations and personality traits are related to side effects reported in active treatment conditions. In cancer patients, for example, pretreatment expectations predicted postchemotherapy nausea independent of prior experience (Roscoe et al, 2000). In an experimental paradigm, manipulation of drug effect information altered side effects in healthy subjects 
treated with a muscle relaxant (carisoprodol) or placebo (Flaten et al, 1999). In relation to personality measures, higher scores on the neuroticism-stability scale of the Eysenck Personality Questionnaire-Revised (Eysenck and Eysenck, 1991) and the Somatasensory Amplification Scale (Barsky et $a l, 1988$ ) were linked to greater side effect reporting among healthy subjects treated with an antidepressant (moclobemide) or placebo (Davis et al, 1995). Personality factors that are related to side effect reporting may have a genetic basis; for example, neuroticism has been linked to variants in the serotonin transporter-linked polymorphic region (5-HTTLPR; Greenberg et al, 2000). Personality factors also may overlap with expectations. Individuals scoring high on neurotic or hypochondriacal traits, for example, may be more likely to expect adverse side effects in an ambiguous situation.

Changes in prefrontal EEG theta have been linked to activity in the anterior cingulate cortex (ACC), an area that has been implicated in the expectation of pain (Sawamoto et $a l, 2000$ ) and unpleasant emotional stimuli (Ueda et al, 2003), and has been shown to have prognostic value for determining clinical response to antidepressant medications. In normal subjects, theta activity recorded from prefrontal surface electrodes has been positively correlated with activity in the ACC as measured by magnetoencephalography (MEG; Asada et al, 1999; Ishii, 1999) and positron emission tomography (PET; Pizzagalli et al, 2003). Changes in prefrontal theta cordance specifically have been associated with perfusion in the rostral ACC (Brodman areas 24 and 32) as measured by PET (Leuchter et al, 2004). In depressed subjects, pretreatment hyperactivation of Brodman areas 24 and 32 as measured by PET (Mayberg et al, 1997) and EEG theta (Pizzagalli et al, 2001) has been associated with antidepressant response. It is possible that the prefrontal placebo lead-in effect found in the present study reflects relationships among ACC activity, expectations and antidepressant medication effects.

Changes in prefrontal cordance during placebo lead-in might have reflected a number of nonpharmacodynamic factors that are associated with the reporting of adverse events, however we did not observe any association between brain functional changes and side effect burden in the placebo group. The lack of a finding in the placebo group may reflect the fact that placebo subjects did not report many side effects; subjects randomized to placebo reported significantly fewer side effects at every postrandomization visit than did those assigned to venlafaxine. The relationship between neurophysiologic changes during placebo lead-in and postrandomization side effect burden in the medication group suggests that brain changes at the end of lead-in may indicate a vulnerability to antidepressant side effects.

Several caveats are warranted in interpreting the present findings. First, it is unknown whether the adverse events reported during venlafaxine administration were actually medication side effects. There is a wide range of known side effects of venlafaxine IR and we did not make any determinations as to which adverse events should be considered 'true' side effects. Future studies could elect to focus on individual side effects of particular interest such as nausea or sexual side effects. Second, there may be important differences between our measure of side effect burden and side effect tolerability. We found that the four venlafaxine subjects who dropped out of the study due to adverse events were not among those in the top quartile of side effect burden. This suggests that factors other than the average number of side effects per visit (such as side effect type or severity) may be responsible for nonadherence. Changes in prefrontal function during placebo lead-in appear to be particularly sensitive to the reporting of side effects and may not reflect likelihood for treatment adherence. Third, our use of normal subjects raises the question of generalizability to clinical populations. Healthy subjects who participate in a study for payment may differ from depressed subjects who stand to benefit from treatment, and, changes in neurophysiologic function may be different between these populations. Lastly, due to the relatively large number of tests and small sample size, results of these exploratory analyses should be considered tentative until replicated. For example, although we corrected for the number of brain regional tests per timepoint, there remains the issue of correcting for multiple comparisons across time-points.

Changes in prefrontal brain function during placebo leadin may indicate a susceptibility to developing adverse symptoms during antidepressant treatment. Future studies should test this hypothesis in depressed subjects. If replicated, these results may add to our understanding of factors that contribute to side effects in the clinical setting.

\section{ACKNOWLEDGEMENTS}

This work was supported by Psychobiology Training Grant T32MH17140 from the National Institute of Mental Health (NIMH) to Dr Hunter (PI, AF Leuchter), by Research Scientist Development Award KO2-MH01165 from the $\mathrm{NIMH}$ to Dr Leuchter, by a Young Investigator Award (Staglin Music Festival Investigator) from the National Alliance for Research in Schizophrenia and Depression (NARSAD) to Dr Morgan, and by Career Development Award KO8-MH01483 from the NIMH to Dr Cook. We also acknowledge the grant support of Lilly Research Laboratories.

\section{REFERENCES}

Ader R (1997). The role of conditioning in pharmacotherapy. In: Harrington A (ed). The Placebo Effect: An Interdisciplinary Exploration. Cambridge University Press: Cambridge, MA. pp 138-165.

Anderson IM, Tomenson BM (1995). Treatment discontinuation with selective serotonin reuptake inhibitors compared with tricyclic antidepressants: a meta-analysis. BMJ 310: 1433-1438.

Asada H, Fukudea Y, Tsunoda S, Yamaguchi M, Tonoike M (1999). Frontal midline theta rhythms reflect alternative activation of prefrontal cortex and anterior cingulate cortex in humans. Neurosci Lett 274: 29-32.

Barge-Schaapveld DQ, Nicolson NA (2002). Effects of antidepressant treatment on the quality of daily life: an experience sampling study. J Clin Psychiatry 63: 477-485.

Barsky AJ, Goodson JD, Lane RS, Cleary PD (1988). The amplification of somatic symptoms. Psychosom Med 50: 510-519. 
Barsky AJ, Saintfort R, Rogers MP, Borus JF (2002). Nonspecific medication side effects and the nocebo phenomenon. JAMA 287: 622-627.

Benedetti F, Pollo A, Lopiano L, Lanotte M, Vighetti S, Rainero I (2003). Conscious expectation and unconscious conditioning in analgesic, motor, and hormonal placebo/nocebo responses. J Neurosci 23: 4315-4323.

Brenner RP, Ulrich RF, Reynolds CF (1995). EEG spectral findings inhealthy elderly men and women-sex differences. Electroencephalogr Clin Neurophysiol 94: 1-5.

Cook IA, Leuchter AF (2001). Prefrontal changes and treatment response prediction in depression. Semin Clin Neuropsychiatry 6: $113-120$

Cook IA, Leuchter AF, Morgan M, Witte E, Stubbeman WF, Abrams $M$ et al (2002). Early changes in prefrontal activity characterize clinical responders to antidepressants. Neuropsychopharmacology 27: 120-131.

Cook IA, Leuchter AF, Uijtedehaage SHJ, Holschneider D, Osato S, Abrams $M$ et al (1998a). Altered cerebral energy utilization in late life depression. J Affect Disord 49: 89-99.

Cook IA, Leuchter AF, Witte E, Abrams M, Uijtdehaage SH, Stubbeman W et al (1999). Neurophysiologic predictors of treatment response to fluoxetine in major depression. Psychiatry Res 85: 263-273.

Cook IA, O'Hara R, Uijtdehaage SH, Mandelkern M, Leuchter AF (1998b). Assessing the accuracy of topographic EEG mapping for determining local brain function. Electroencephalogr Clin Neurophysiol 107: 408-414.

Davis C, Ralevski E, Kennedy SH, Neitzert C (1995). The role of personality factors in the reporting of side effect complaints to moclobemide and placebo: a study of healthy male and female volunteers. J Clin Psychopharmacol 15: 347-352.

Eysenck HJ, Eysenck SBG (1991). Manual of the Eysenck Personality Scales. Hodder \& Stoughton: London.

First MB, Spitzer RL, Gibbom M, Williams J (1994). Structured Clinical Interview for Axis I DSM-IV Disorders-Patient Edition (SCID-I/P, version 2.0). Biometrics Research Department, NY State Psychiatric Institute: New York.

Flaten MA, Simonsen T, Olsen H (1999). Drug-related information generates placebo and nocebo responses that modify the drug response. Psychosom Med 61: 250-255.

Gandhi TK, Weingart SN, Borus J, Seger AC, Peterson J, Burdick E et al (2003). Adverse drug events in ambulatory care. $N$ Engl J Med 348: 1556-1564.

Greenberg BD, Li Q, Lucas FR, Hu S, Sirota LA, Benjamin J et al (2000). Association between the serotonin transporter promoter polymorphism and personality traits in a primarily female population. Am J Med Genet 96: 202-216.

Greer MM, Kremer C, Rodrigues HE, Schatzberg AF (2003). Pharmacogenetics of antidepressant medication intolerance. $\mathrm{Am}$ J Psychiatry 160: 1830-1835.

Hahn RA (1997). The nocebo phenomenon: concept, evidence, and implications for public health. Prev Med 26: 607-611.

Hamilton M (1960). A rating scale for depression. J Neurol Neurosurg Psychiatry 23: 56-62.

Ishii R, Shinosaki K, Ukai S, Inouye T, Ishihara T, Yoshimine T et al (1999). Medial prefrontal cortex generates frontal midline theta rhythm. Neuroreport 10: 675-679.

Jewett DL, Fein G, Greenberg MH (1990). A double-blind study of symptom provocation to determine food sensitivity. $N$ Engl J Med 323: 429-433.

Kennedy WP (1961). The nocebo reaction. Medical World News 91: 203-205.

Kissel P, Barrucand D (1964). Placebos and Placebo Effect in Medicine (Placebos et effet placebo en medecine). Masson: Oxford, England, 240pp.

Lessard E, Yessine MA, Hamelin BA, O'Hara G, LeBlanc J, Turgeon J (1999). Influence of CYP2D6 activity on the disposition and cardiovascular toxicity of the antidepressant agent venlafaxine in humans. Pharmacogenetics 9: 435-443.

Leuchter AF, Cook IA, Greenwald SD, Kofol T (2004). Prefrontal EEG cordance correlates with anterior cingulate perfusion. Poster Presented at the Annual Meeting of the American Psychiatric Association, NR671.

Leuchter AF, Cook IA, Witte EA, Morgan M, Abrams M (2002). Changes in brain function of depressed subjects during treatment with placebo. Am J Psychiatry 159: 122-129.

Leuchter AF, Newton TF, Cook IA, Walter DO, RosenbergThompson S, Lachenbruch PA (1992). Changes in brain functional connectivity in Alzheimer's-type and infarct dementia. Brain 115: 1543-1561.

Leuchter AF, Uijtdehaage SHJ, Cook IA, O'Hara R, Mandelkern M (1999). Relationship between brain electrical activity and cortical perfusion in normal subjects. Psychiatry Res Neuroimaging 90: 125-140.

Mayberg HS, Brannan SK, Mahurin RK, Jerabek PA, Brickman JS, Tekell JL et al (1997). Cingulate function in depression: a potential predictor of treatment response. Neuroreport 8: 1057-1061.

Mayberg HS, Silva JA, Brannan SK, Tekell JL, Mahurin RK, McGinnis S et al (2002). The functional neuroanatomy of the placebo effect. Am J Psychiatry 159: 728-737.

McFadden Jr ER, Luparello T, Lyons HA, Bleecker E (1969). The mechanism of action of suggestion in the induction of acute asthma attacks. Psychosom Med 31: 134-143.

Montgomery SA, Henry J, McDonald G, Dinan T, Lader M, Hindmarch I et al (1994). Selective serotonin reuptake inhibitors: meta-analysis of discontinuation rates. Int Clin Psychopharmacol 9: 47-53.

Montgomery SA, Kasper S (1995). Comparison of compliance between serotonin reuptake inhibitors and tricyclic antidepressants: a meta-analysis. Int Clin Psychopharmacol Suppl 4: 33-40.

Myers MG, Cairns JA, Singer J (1987). The consent form as a possible cause of side effects. Clin Pharmacol Ther 42: 250-253.

Ohayon MM, Schatzberg AF (2003). Using chronic pain to predict depressive morbidity in the general population. Arch Gen Psychiatry 60: 39-47.

Pizzagalli D, Pascual-Marqui RD, Nitschke JB, Oakes TR, Larson $\mathrm{CL}, \mathrm{Abercrombie} \mathrm{HC}$ et al (2001). Anterior cingulate activity as a predictor of degree of treatment response in major depression: evidence from brain electrical tomography analysis. $A m \mathrm{~J}$ Psychiatry 158: 405-415.

Pizzagalli DA, Oakes TR, Davidson RJ (2003). Coupling of theta activity and glucose metabolism in the human rostral anterior cingulate cortex: an EEG/PET study of normal and depressed subjects. Psychophysiology 40: 939-949.

Roberts RL, Joyce PR, Mulder RT, Begg EJ, Kennedy MA (2002). A common P-glycoprotein polymorphism is associated with nortriptyline-induced postural hypotension in patients treated for major depression. Pharmacogenomics 2: 191-196.

Roscoe JA, Hickok JT, Morrow GR (2000). Patient expectations as predictor of chemotherapy-induced nausea. Ann Behav Med 22: 121-126.

Rosenzweig P, Brohier S, Zipfel A (1993). The placebo effect in healthy volunteers: influence of experimental conditions on the adverse events profile during phase I studies. Clin Pharmacol Ther 54: 578-583.

Rush AJ (2001). Sequenced treatment alternatives to relieve depression $\left(\mathrm{STAR}^{\star} \mathrm{D}\right)$ protocol. NIMH Contract GMO-010111. http://www.edc.gsph.pitt.edu/stard/.

Sawamoto N, Honda M, Okada T, Hanakawa T, Kanda M, Fukuyama $\mathrm{H}$ et al (2000). Expectation of pain enhances responses to nonpainful somatosensory stimulation in the anterior cingulate cortex and parietal operculum/posterior insula: an event-related functional magnetic resonance imaging study. Neuroscience 20: 7438-7445. 
Siegel S (2002). Explanatory mechanisms for placebo effects: Pavlovian conditioning. In: Guess HA, Kleinman A, Kusek JW, Engel LW (eds). The Science of the Placebo: Toward an Interdisciplinary Research Agenda. BMJ Books: London. pp 133-157.

Ueda K, Okamoto Y, Okada G, Yamashita H, Hori T, Yamawaki S (2003). Brain activity during expectancy of emotional stimuli: an fMRI study. Neuroreport 14: 51-55.
Ulrich G, Haug HJ, Fahndrich E (1994). Acute versus chronic EEG effects of maprotiline- and in clomipramine-treated depressive inpatients and the prediction of therapeutic outcome. $J$ Affect Disord 32: 213-217.

Yoshida K, Naito S, Takahashi H, Sato K, Ito K, Kamata M et al (2003). Monoamine oxidase A gene polymorphism, 5-HT 2A receptor gene polymorphism and incidence of nausea induced by fluvoxamine. Neuropsychobiology 48: 10-13. 